\title{
Development of Multi-beam Long Trace Profiler
}

\author{
Kiranmayee Kilaru ${ }^{1}$, Daniel J. Merthe ${ }^{2}$, Zulfiqar Ali ${ }^{2}$, Mikhail V. Gubarev ${ }^{1}$, Thomas Kester ${ }^{1}$, \\ Wayne R. McKinney ${ }^{2}$, Peter Z. Takacs ${ }^{3}$ and Valeriy V. Yashchuk ${ }^{2}$ \\ ${ }^{1}$ NASA Marshall Space Flight Center, Huntsville, AL 35812, USA \\ ${ }^{2}$ Lawrence Berkeley National Laboratory, Berkeley, CA 94720, USA \\ ${ }^{3}$ Brookhaven National Laboratory, Upton, NY, 11973, USA
}

\begin{abstract}
In order to fulfill the angular resolution requirements and make the performance goals for future NASA missions feasible, it is crucial to develop instruments capable of fast and precise figure metrology of x-ray optical elements for further correction of the surface errors. The Long Trace Profilometer (LTP) is an instrument widely used for measuring the surface figure of grazing incidence X-ray mirrors. In the case of replicated optics designed for x-ray astronomy applications, such as mirrors and the corresponding mandrels have a cylindrical shape and their tangential profile is parabolic or hyperbolic. Modern LTPs have sub-micro radian accuracy, but the measuring speed is very low, because the profilometer measures surface figure point by point using a single laser beam. The measurement rate can be significantly improved by replacing the single optical beam with multiple beams. The goal of this study is to demonstrate the viability of multi-beam metrology as a way of significantly improving the quality and affordability of replicated $\mathrm{x}$-ray optics. The multi-beam LTP would allow one- and twodimensional scanning with sub-micro radian resolution and a measurement rate of about ten times faster compared to the current LTP. The design details of the instrument's optical layout and the status of optical tests will be presented.
\end{abstract}

Keywords: Long Trace Profiler, optical metrology, x-ray optics metrology, multi-beam profiler

\section{About LTP}


The long trace profilometer (LTP) is a slope-measuring instrument widely used for measuring surface-figure of grazing incidence X-ray optics. The achievable angular resolution with such optics is about 10 arcseconds with the present state of art technology. There is an increasing demand for the improved resolution with these optics and one of the points that dictates the quality of the optics is the quality of metrology. Full-shell wolter-type grazing incidence optics are regularly fabricated at MSFC using electroformed replication process. In this process X-ray mirrors/shells are replicated off a figured and super-polished mandrel. MSFC has an in-house-built LTP (Misha's calibration paper), which is used to measure the profiles of outer surface of mandrels, and the inner surface of the replicated X-ray shells. The LTP uses a visible laser beam to scan along the test surface, which records the slope difference between adjacent scan points. This slope gradient data is then converted to height data that gives a measure of the profile of test surface along the axial length. Multiple azimuthal measurements give the 2D topographical data of the surface under test. The slope resolution possible with this system is $<1 \mu \mathrm{rad}$. Time taken to measure a surface of $\sim 300 \mathrm{~mm}$ length is approximately 5 minutes and typically three measurements are made along each axial position, which are then averaged to nullify any random noises in the metrology process. Therefore, the time taken to measure and process a single axial scan for such a surface is about 15 minutes, which if minimized can help in making the mirror fabrication process more cost- and time-efficient. Using multiple laser beams, to scan the test surface, in the place of a single beam would be an innovative approach to achieve this. The development of this multi-beam LTP as a proof-of-concept study is currently underway at MSFC in collaboration with Lawrence Berkeley National Labs and Brookhaven National labs.

\section{Multi-beam LTP}

Multi-beam LTP is believed to provide a significant improvement in the measurement rate compared to a single-beam LTP. Point-by-point measurement done with the single beam LTP can be replaced by simultaneous measurement of a section of surface using multiple beams 
that are processed in a single instance. Figure1 shows the geometrical layout of the metrology approach using multiple laser beams.

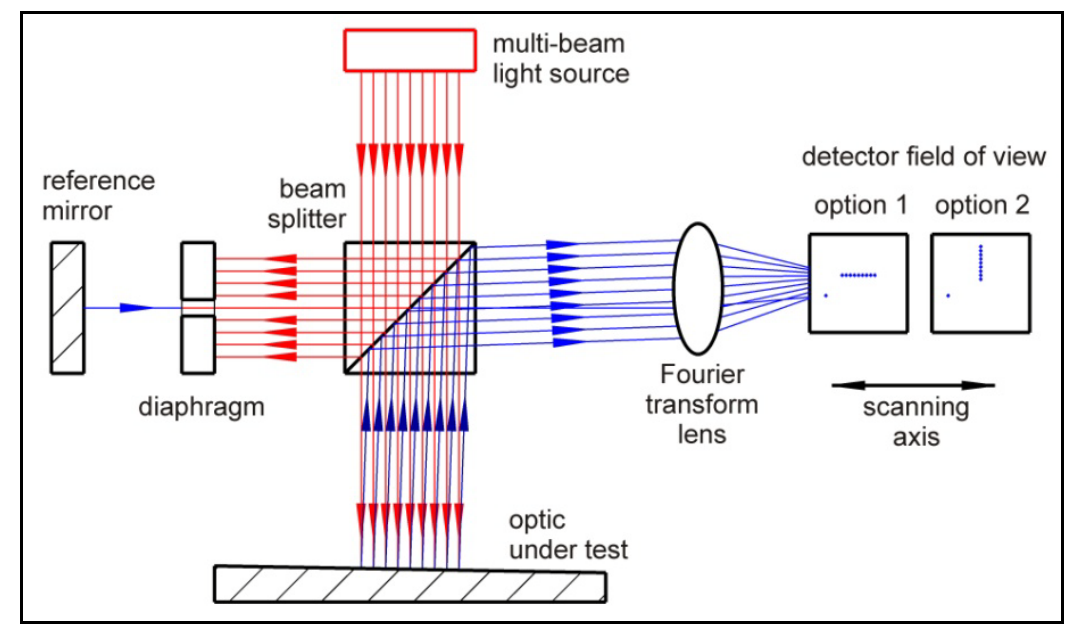

Figure 1: Geometrical layout of multi-beam LTP

A laser beam is split into multiple equal intensity beams which when pass through a polarizing beam splitter get divided into two segments. One of these is directed towards a reference beam and the other to the test surface. Reflected beams off from each of these two segments are then focused onto a 2D detector using a Fourier-Transform/F-Theta (FT) lens. Study has been made to analyze the possible geometrical layouts, and technical requirements and specifications for components such as multiple-beam generator, FT lens, polarizing beam splitter and 2D detector. Table 1 shows the typical specifications formulated for the multiple optical beams.

\begin{tabular}{|c|c|c|}
\hline Parameter & Value & Comments \\
\hline Number of (Gaussian) beams, $n$ & $5-10$ & $n \approx$ size of the LTP optics $/ \Delta x$ \\
\hline
\end{tabular}




\begin{tabular}{|c|c|c|}
\hline Single beam spot size (Gaussian), $t$ & $2.1 \mathrm{~mm}$ & at $1 / e^{2}$ level for FC5 collimator [11] \\
\hline Single beam divergence, $\alpha$ & $0.2 \mathrm{mrad}$ & from the spot size on the LTP detector \\
\hline Single beam pointing stability, $\delta \alpha$ & $0.1 \mu \mathrm{rad}$ & provided by fiber-coupled diode laser [12] \\
\hline Spatial separation of adjacent beams, $\Delta x$ & $1-2 \mathrm{~mm}$ & desired separation of measured points on optic \\
\hline Angular separation of adjacent beams, $\beta$ & $0.25 \mathrm{mrad}$ & $\beta \geq \alpha$ to avoid spurious interference \\
\hline Stability of angular separations, $\Delta \beta$ & $0.1 \mu \mathrm{rad}$ & to be experimentally verified \\
\hline Max-min variation of intensities, $\Delta I$ & $\pm 0.05 I_{0}$ & $I_{0}=(0.3-1) \mathrm{mW}$ \\
\hline
\end{tabular}

Table1: Requirements of a multi-beam light source

\section{FT lens and 2D detector}

The conventional LTP is a pencil beam interference in which each point of scan has two beams which interfere and this interference pattern is focused onto the detector using the FT lens. The detector used with the current LTP is a linear detector array with the pixel size of 25 $\mu \mathrm{m} \times 2.5 \mathrm{~mm}$. The detector software used for data processing identifies the minima of the interference pattern to detect the location of the beam on the detector. This can be replaced with a stable single beam laser if the maxima of the peak can be read-out with desired accuracies. Also, the present LTP uses an FT lens of focal length 1 meter, which means the distance from the FT lens to the detector is 1 meter. Therefore, the optical configuration uses folding mirrors to redirect and fold the beam. As in any optical design additional optical components add up to the sources of error. These folding mirrors can be avoided in the new configuration if an FT lens of small focal length can be used. But, the focal length of the FT lens, together with the detector's positional accuracy specification, defines the resolution possible with the detector as shown in equation 1 
$\alpha=\frac{d}{2 * E F L}$

The positional accuracy is typically a fraction of the pixel size and depends on several factors such as non-constant pixel spacing of the detector, detector-noise, non-uniformity in the detector response and the stability of the laser source. The smaller the pixel size, the better is the resolution of the LTP system. Assuming the positional accuracy of 0.03 times the pixel size, a $500 \mathrm{~mm}$ to $700 \mathrm{~mm}$ focal length lens when used in combination of $8 \mu \mathrm{m}$ pixel size detector, would result in the system resolution of better than $0.25 \mu$ rad as shown in Table 2 .

\begin{tabular}{|r|c|c|c|c|c|}
\hline \multicolumn{1}{|l|}{$\mathrm{fl} \mathrm{mm}$} & 500 & 550 & 600 & 700 & 800 \\
\hline pixel $(\mu \mathrm{m})$ & & & & & \\
\hline 4 & 0.12 & 0.11 & 0.10 & 0.09 & 0.08 \\
\hline 8 & 0.24 & 0.22 & 0.20 & 0.17 & 0.15 \\
\hline 10 & 0.30 & 0.27 & 0.25 & 0.21 & 0.19 \\
\hline 14 & 0.42 & 0.38 & 0.35 & 0.30 & 0.26 \\
\hline 18 & 0.54 & 0.49 & 0.45 & 0.39 & 0.34 \\
\hline
\end{tabular}

Table 2. Resolution in microradians calculated for differet combinations of $\mathrm{Ft}$ lens focal length and detector pixel size.

The detector also has a requirement of wide area of about $20 \mathrm{~mm} \times 20 \mathrm{~mm}$ so as to be able to accommodate multiple beams number of beams along one plane while being able to record the beam translation due to slope errors on the other. Comparative study of the available detectors has been made and $1^{\text {st }}$ vision's JAI AM 1600 GE camera has been chosen which has a $7.4 \mu \mathrm{m}$ pixel size with $36.1 \times 24 \mathrm{~mm}$ of active area.

The FT lens of $500 \mathrm{~mm}$ diameter has been custom designed for the application. The design is challenging given to the requirements such as low distortion in order to minimize the effect of the lens on systematic errors; wide lateral range required to accommodate multiple beams and the large angular range of the surface slope that is desired to be measured. The final design is an air-spaced doublet as shown in Figure 2. Alternative lens designs were 
considered such as cemented doublet, cemented triplet and an air-spaced triplet. The desired performance could not be achieved with a cemented doublet and the triplet lenses were not necessary.

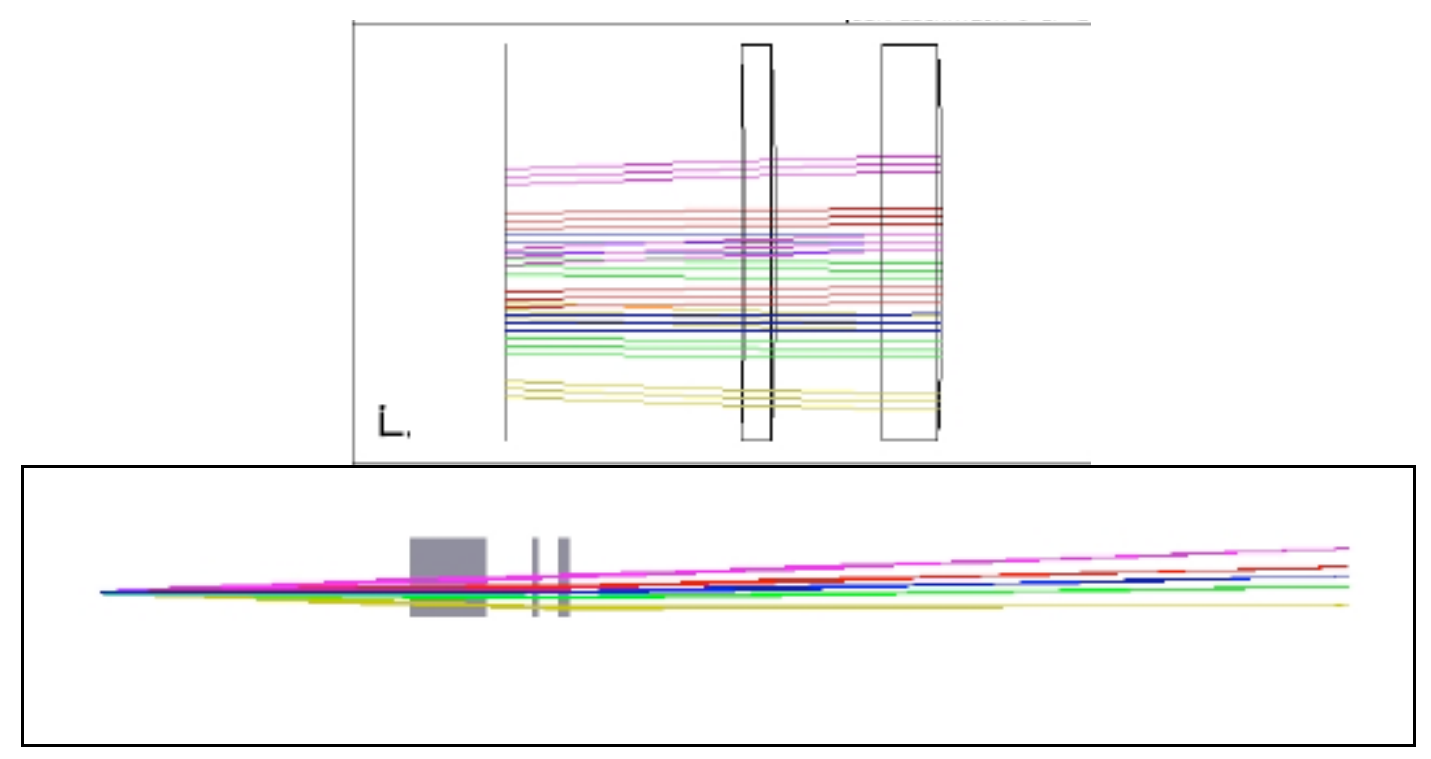

Figure 2. Fourier Transform lens design

This lens has recently been fabricated and been tested for the surface RMS roughness. The data of the transmitted wavefront error of 3 sample regions were provided by the manufacturers. Low-frequency figure error was removed from this data leaving the residual surface that contains higher-spatial frequencies. A 2D PSD is calculated for this residual surface using a conventional DFT. A radial PSD function is calculated by integrating the values over all azimuths, from which RMS roughness over the $5 \mathrm{~mm}$ - $100 \mu \mathrm{m}$ bandwidth range is calculated to be $0.38 \mathrm{~nm}$. Therefore, the tested component meets the required specification of $<0.5 \mathrm{~nm}$ over the specified bandwidth range.

\section{Multiple-beam generation}


One of the major challenges in the development of multi-beam LTP is the generation of multiple optical beams. These beams, ideally, need to have equal intensity with the defined divergence between individual beams. To achieve this, a parallel plate glass plate / etalon with a small wedge is considered. The amount of wedge is defined based on the divergence value between the beams. The etalon has a custom coating on one of the planes so as to obtain equal intensity beams. The central region of the other plane of the etalon has a coating with $100 \%$ reflection as shown in Figure 3.

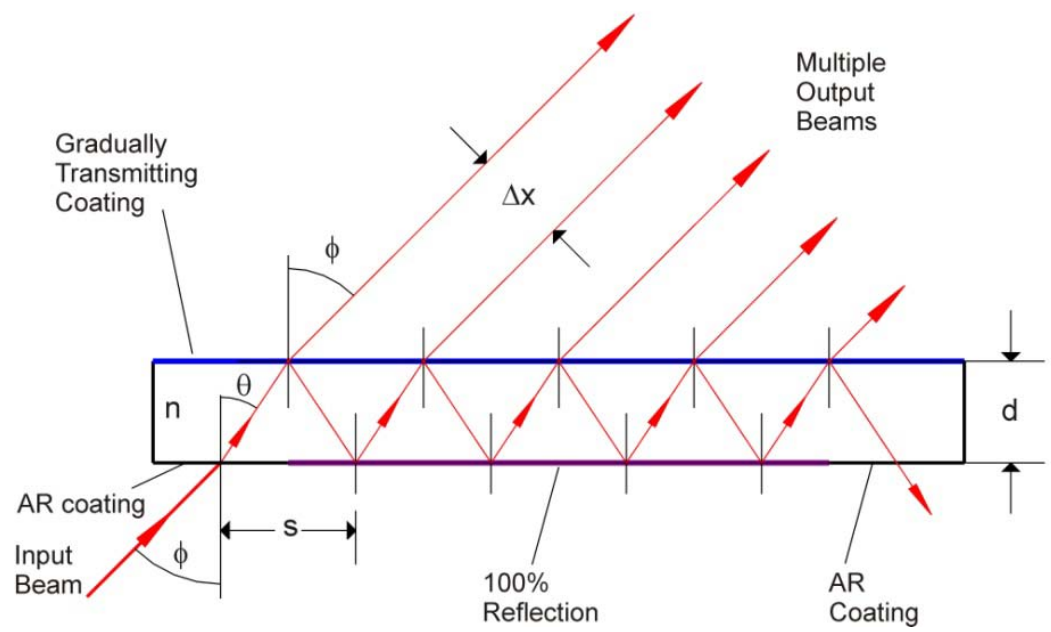

Figure 3. Geometrical layout of multiple-beam splitting etalon

Such beam splitters have been used for wafer curvature measurements [2], for strain monitoring in thin films [3], as well as to produce multiple, equal-intensity beams for twodimensional beam steering [4] and for a galvanometric scanner of a confocal scanning microscope [5].

The etalon will have $50 \mathrm{~mm} \times 50 \mathrm{~mm}$ dimension with a thickness of $3 \mathrm{~mm}$ and a wedge angle of 60 radian. Analysis has been done to study the possible approaches for the custom coating on the etalon. The performed analysis has shown that a pattern of discrete coatings, each with the optimal constant reflectivity, is the most cost efficient. The geometry of such an 
approach is shown in Figure 4 with the reflectivity and transmittance values of each zone mentioned in Table 3.

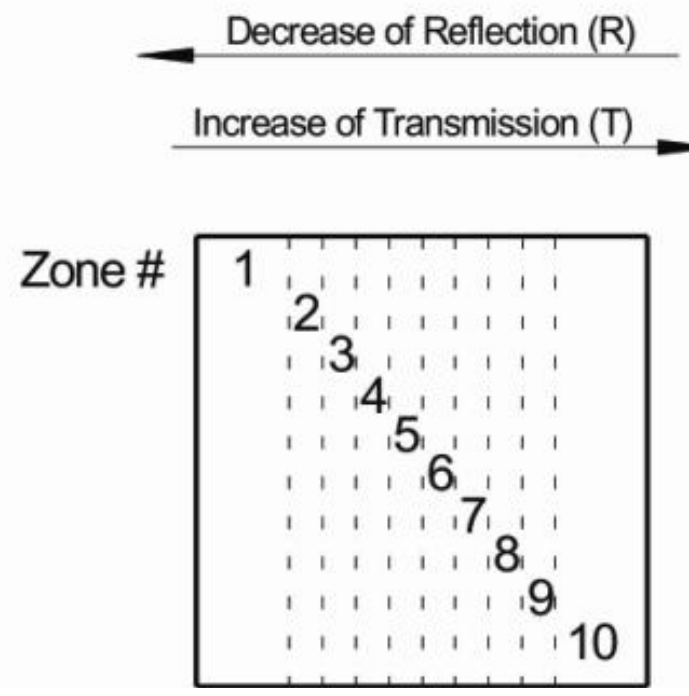

Figure 4. Discrete coating approach

\begin{tabular}{|l|c|c|c|c|c|c|c|c|c|c|}
\hline Zone & 1 & 2 & 3 & 4 & 5 & 6 & 7 & 8 & 9 & 10 \\
\hline $\mathrm{R}_{\mathrm{Z}}, \%$ & 93.33 & 92.86 & 92.31 & 91.67 & 90.91 & 90.00 & 88.89 & 87.50 & 85.71 & 83.33 \\
\hline $\mathrm{T}_{\mathrm{Z}}, \%$ & 6.67 & 7.14 & 7.69 & 8.33 & 9.09 & 10.00 & 11.11 & 12.50 & 14.29 & 16.67 \\
\hline
\end{tabular}

Table 3. Reflectance and transmittance values of discrete coating zones.

The discrete coating can be designed to provide $100 \%$ efficiency of usage of the laser beam intensity. Moreover, the coating will not perturb the beam shape unless the beam spot is totally inside the specified area. A drawback of the discrete coating is the lack of possibility for tuning the multiple-beam splitter geometry.

A continuous coating with an optimal reflectivity distribution is impractical. Accordingly, we have analyzed the expected performance of a continuous coating, which is a linear 
approximation to the ideal one. It is possible to design a coating with linearly distributed reflectivity that would provide an array of 10 beams with intensity variation below $10 \%$. However, with such coating, the efficiency of usage of the laser beam intensity would be on the level of $\sim 30 \%$.

\section{Other details}

A polarizing cube beam splitter is used to split the laser beams into two paths - one to the surface under test and the other to the reference surface. Ideally, this component could be custom made to the desired surface accuracies so as to avoid any systematic errors, but budget limitations limited us to buy an off-the shelf component. The size of the beam splitter is $50 \mathrm{~mm}$ $x 50 \mathrm{~mm}$. This beam splitter has recently been purchased and surface characterizing test are underway using Zygo.

The software interface for the new 2D detector is being developed. The initial program to detect the minima of the interference for a total number of 3 beams has been provided by collaborators from Lawrence Berkeley Labs. This program is being modified for the new detector, multiple beams and also as discussed previously to detect the maxima of the optical beam. The tests to check the speed of processing and the stability of the scan are under progress. The initial trials show a speed of $0.5 \mathrm{fps}$ for full frame reading which can further be improved with partial frame scanning.

\section{Conclusion}

A multi-beam LTP is under development at MSFC in collaboration with Lawrence Berkeley National labs and Brookhaven National Labs. This LTP unlike the conventional single beam LTP uses multiple beams to make simultaneous measurements in a single instance along a section of the test surface. This helps in significantly increasing the rate at which surface profile metrology can be done on grazing incidence optics. The design considerations and the 
challenges in the development of such metrology equipment are discussed. Also, the present status with the development of this multi-beam long trace profiler is presented in this paper.

\section{References}

1. "Calibration of vertical-scan long trace profilometer at MSFC", Mikhail Gubarev, Thomas Kester, Peter Takacs, Optical manufacturing and testing IV, Proceedings of SPIE Vol. 4451 (2001)

2. M.M. Rajadhyaksha and R. H. Webb, "Plate Beamsplitter to Produce Multiple EqualIntensity Beams," Appl. Opt. 34(34), 8066-8066 (1995)

3. D. Litvinov, R. Clarke, C. A. Taylor II, D. Barlett, Real-time strain monitoring in thin film growth: cubic boron nitride on Si (100), Mater. Sci. and Eng. B66, 79-82 (1999)

4. M. Toyoshima, F. Fidler, M. Pfennigbauer, and W. R. Leeb, "Two-dimensional optical beam deflector operated by wavelength tuning," Opt. Exp. 14(9) 4092-100 (2006)

5. http://refractiveindex.info/?group=GLASSES\&material=F SILICA 


\section{Development of a Multi-beam Long Trace Profiler}

Kiranmayee Kilaru', Daniel J. Merthe ${ }^{2}$, Zulfiqar Ali², Mikhail V. Gubarev', Thomas Kester', Wayne R. McKinney², Peter Z. Takacs $^{3}$, Valeriy V. Yashchuk ${ }^{2}$

'NASA Marshall Space Flight Center 2Lawrence Berkeley National Laboratory ${ }^{3}$ Brookhaven National Laboratory 


\section{Grazing incidence optics}

Astronomical Applications
HERO

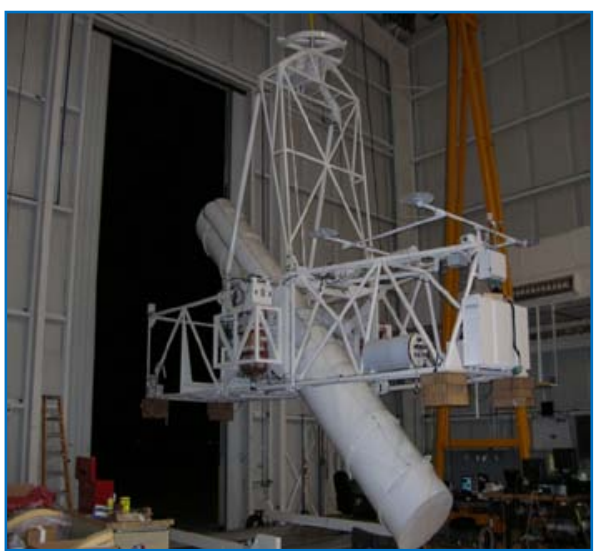

FOXSI

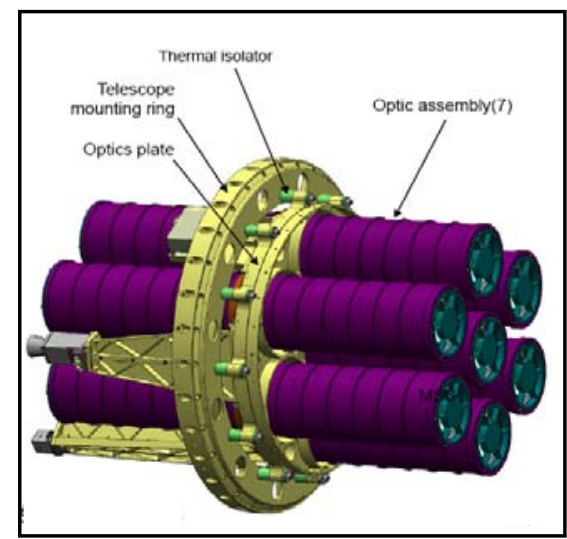

-X-ray mirror - 10 arc secs

- Future optics - increased optical resolution - better quality and efficient metrology

-Quality metrology $\rightarrow$

Quality Optics

Neutron imaging

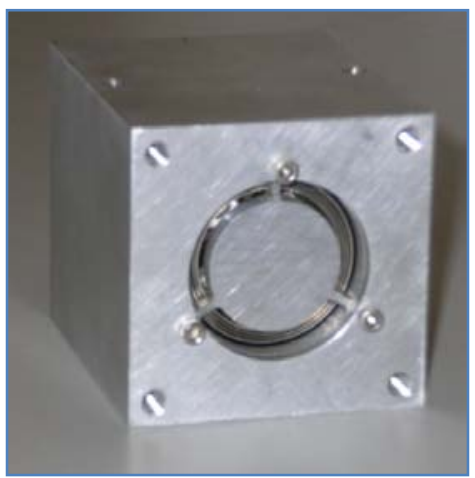

- Multiple large area shells faster metrology - saves time and money 


\section{Long Trace Profilometer}

\section{-Pencil beam interferometry}

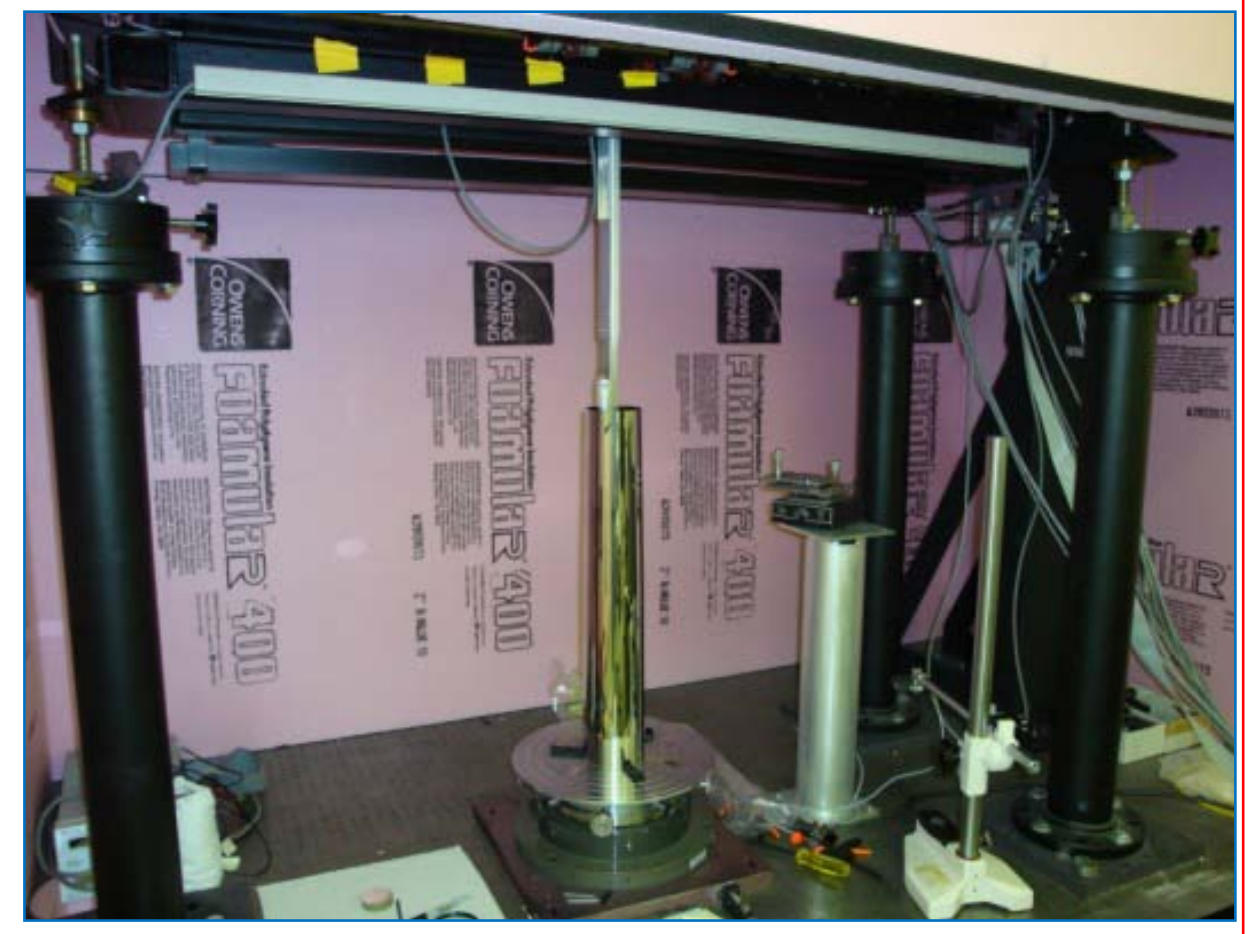

- Measure spatial wavelengths starting from $1 \mathrm{~mm}$

- Laser beam scans point-by- point - slope data

- Uses 2 beam interference

-Position of the minima at the detector - direct measure of the slope

-Accuracies possible $<1$ urad

(2) Time taken to measure is about 5 mins for $300 \mathrm{~mm}$ sample length

-Multiple measurements - 2D topography 


\section{Looking forward..}

- Make use of advanced technology

- Higher resolution 2D detectors

- Stable optical sources

- Increase the speed \& accuracies of measurements

- Higher density data - complete information of mandrel or shell

- Multiple beams - simultaneous measurements? 


\section{Multi-beam LTP}

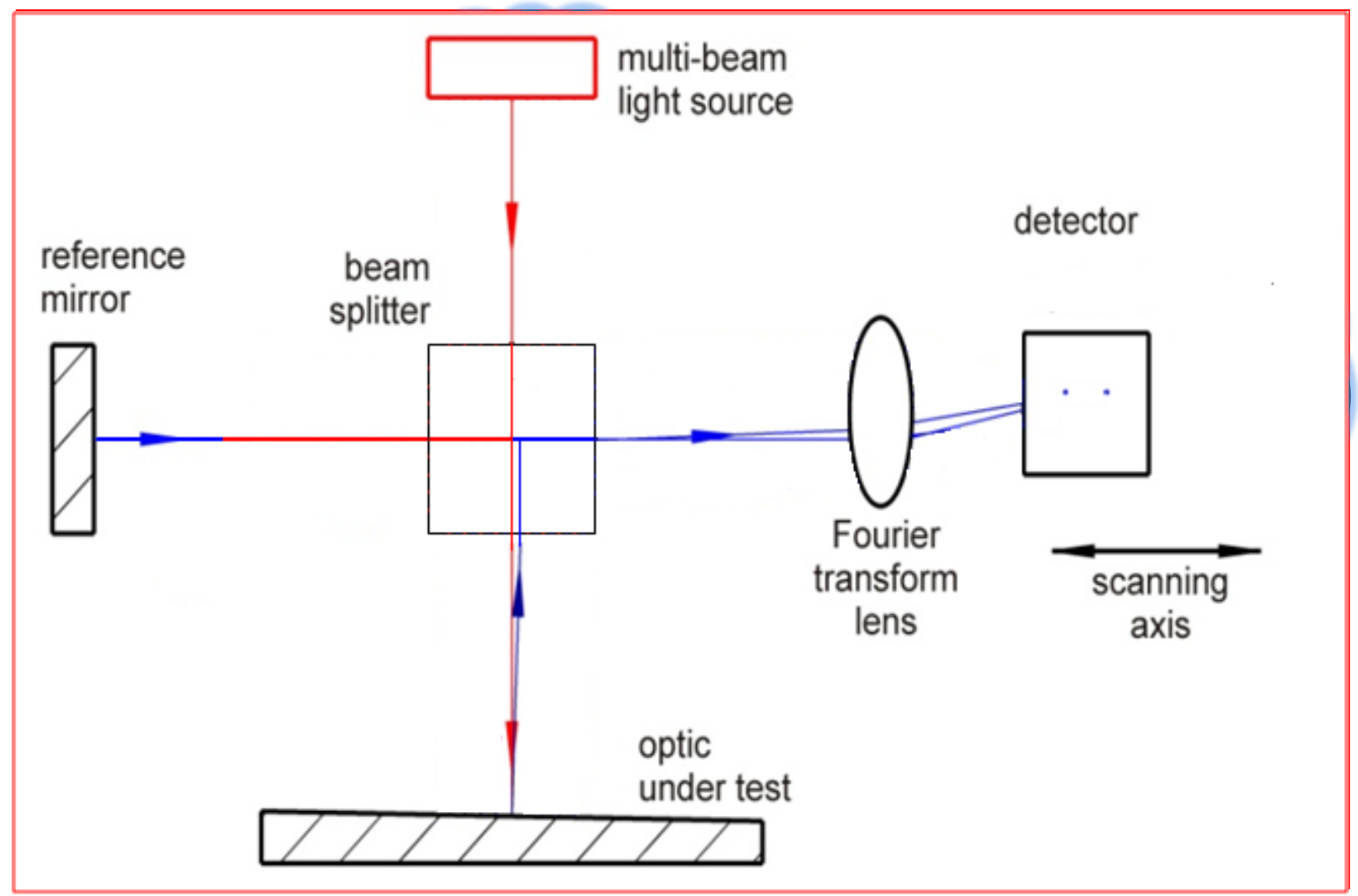

-Proof-of-concept

- To study the limitations of the approach
- Number of beams - 5 to 10

-Spatial separation of beams - 1 to $2 \mathrm{~mm}$ 


\section{Multi-beam LTP Requirements}

- Target - decrease the time of measurement - reasonable accuracy

- Existing systems

- linear array detector - $25 \mu \mathrm{m} \times 2.5 \mathrm{~mm}$ pixel size

- $1 \mathrm{~m}$ focal length FT lens

- Lens and detector $\rightarrow 25 \mu \mathrm{rad}$

- $2 D$ detector

- multiple beams in one plane and beam translation on other plane

- + /- $15 \mathrm{mrad}-20 \mathrm{~mm} \times 20 \mathrm{~mm}$ area

- $8 \mu m$ pixel size

\begin{tabular}{|r||c|c|c|c|c|}
\hline fl $(\mathrm{mm})$ & 500 & 550 & 600 & 700 & 800 \\
\hline pixel $(\mu \mathrm{m})$ & & & & & \\
\hline 4 & 0.12 & 0.11 & 0.10 & 0.09 & 0.08 \\
\hline 8 & 0.24 & 0.22 & 0.20 & 0.17 & 0.15 \\
\hline 10 & 0.30 & 0.27 & 0.25 & 0.21 & 0.19 \\
\hline 14 & 0.42 & 0.38 & 0.35 & 0.30 & 0.26 \\
\hline 18 & & & & & \\
\hline 18 & 0.54 & 0.49 & 0.45 & 0.39 & 0.34 \\
\hline
\end{tabular}




\section{D CCD detector \& FT lens}

- Detector Procured

$-36 \mathrm{~mm} \times 24 \mathrm{~mm}$ area

$-7.4 \times 7.4 \mu \mathrm{m}$ pixel size

$-3.04 \mathrm{fps}$

- Custom designed FT lens

- $500 \mathrm{~mm}$ focal length

- Low distortion - minimize the effects of lens on systematic errors

- Less number of elements - two element system 


\section{Multiple beam generation}

- Multiple beams of equal intensity

- Spatial separation (2.4 mm)

- Angular separation (250 urad)

- Wedged etalon approach

- Customized coating on one side

- $100 \%$ reflection coating on the other

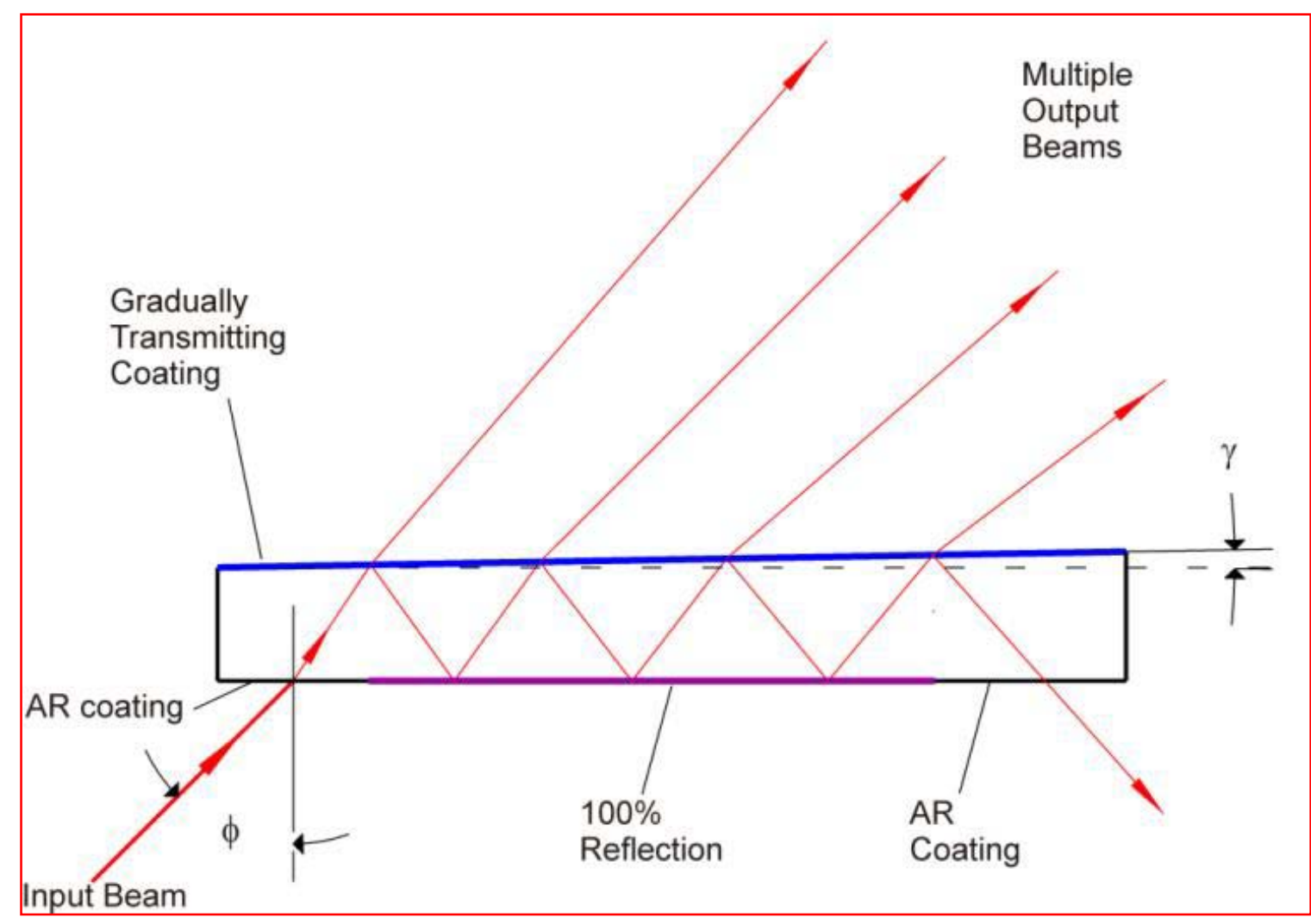

- Dimension-50 $550 \times 3 \mathrm{~mm}$

- Wedge-60 $\mu \mathrm{rad}$ 


\section{Coating - wedged etalon}

Possible approaches

- Continuous ideal gradient coating

- Linear approximation to ideal

-Multilayers

- Discrete coatings - each with constant reflectivity

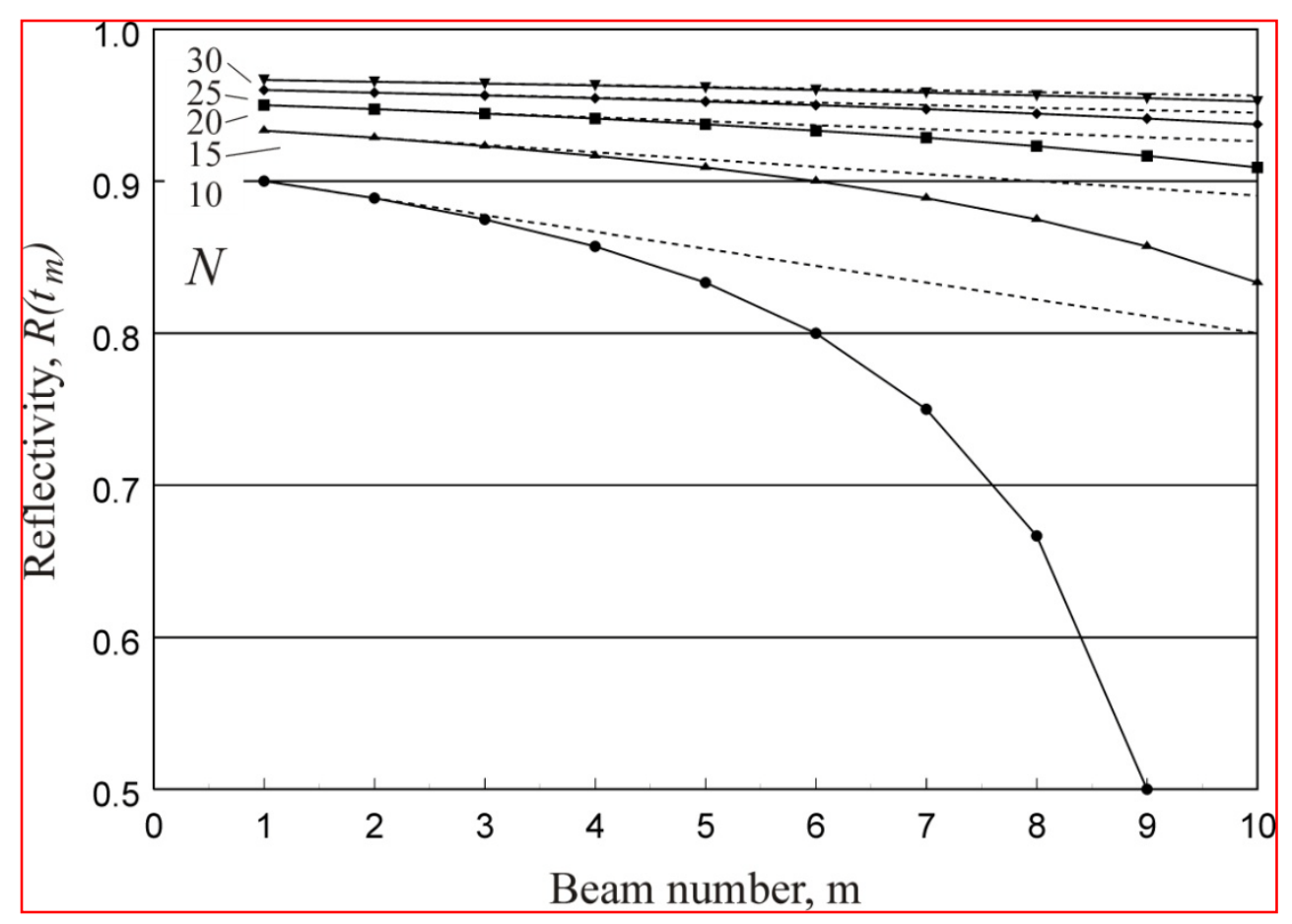




\section{Multiple beam generation - wedged etalon}

- Silver coating with step reflectivity approach

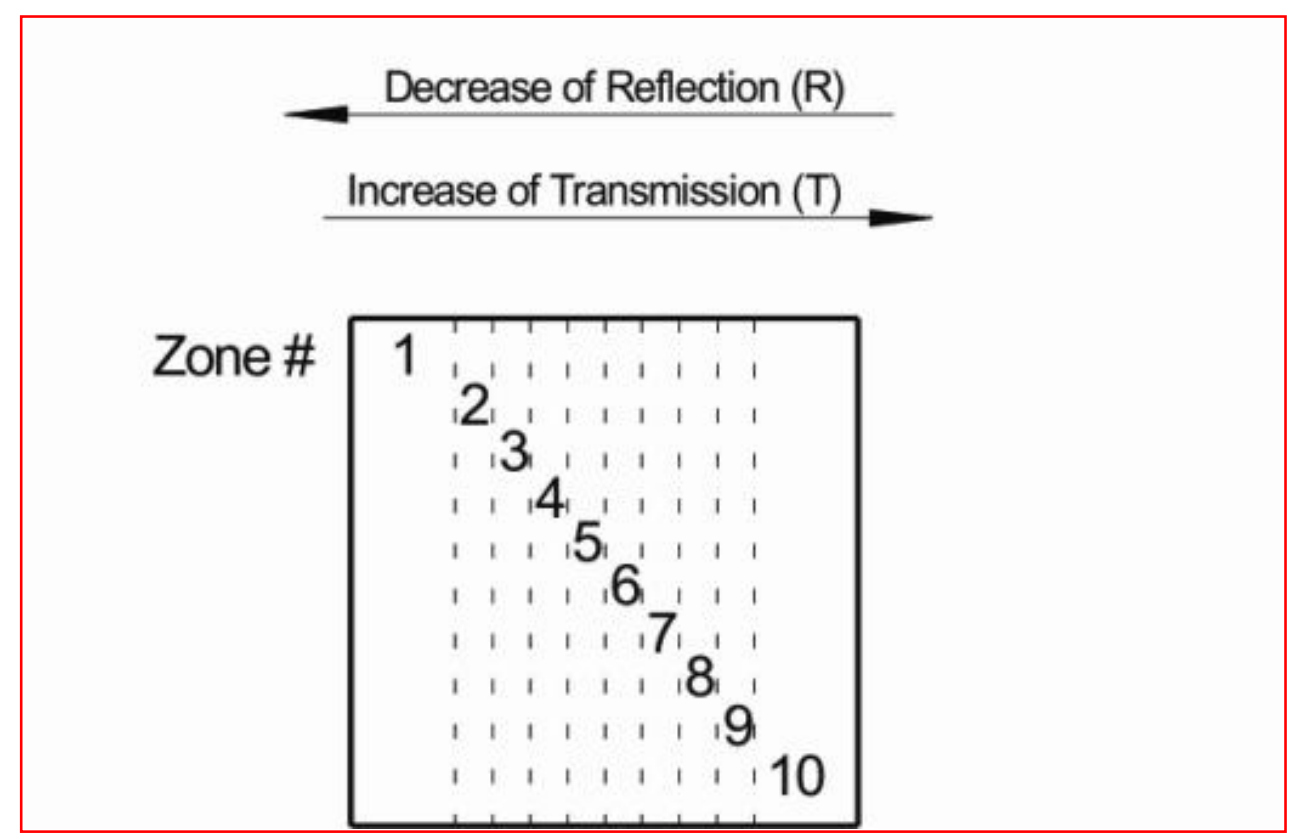

\begin{tabular}{|l|c|c|c|c|c|c|c|c|c|c|}
\hline Zone & 1 & 2 & 3 & 4 & 5 & 6 & 7 & 8 & 9 & 10 \\
\hline $\mathrm{R}_{\mathrm{Z}} \%$ & 93.33 & 92.86 & 92.31 & 91.67 & 90.91 & 90.00 & 88.89 & 87.50 & 85.71 & 83.33 \\
\hline $\mathrm{T}_{\mathrm{Z}} \%$ & 6.67 & 7.14 & 7.69 & 8.33 & 9.09 & 10.00 & 11.11 & 12.50 & 14.29 & 16.67 \\
\hline
\end{tabular}




\section{Cube beamsplitter}

Surface/帕avefront Map

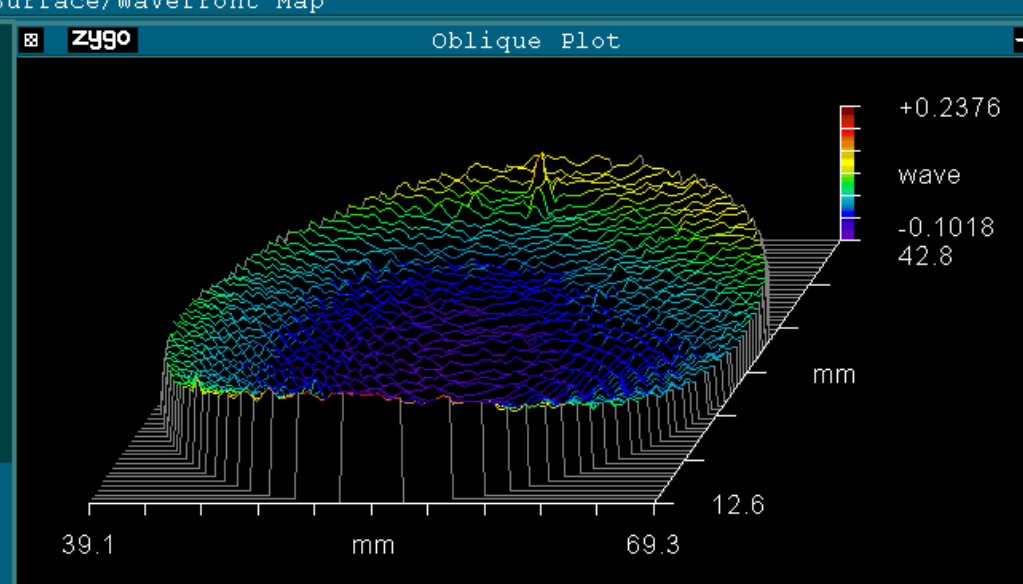

Removed: PST TLT

Trimmed: 0

Aperture OD (\%):

Aperture TD

Filter: off

$$
\text { Q Zygo slope Mag }
$$
4
-Purchased $50 \mathrm{~mm} \times 50 \mathrm{~mm}$ beam splitter tested with zygo for wavefront error

-Analysis of test result in progress

\begin{tabular}{|c|c|c|}
\hline Measurement & Attributes & 」 \\
\hline Wed Jul 20 15: 12:31 2011 & Data Sign: & \\
\hline P/N： TIP 2010 & Scale Factor: 1.00 & \\
\hline S/N: BS CUBE \#2 & Camera Res: & $\mu \mathrm{m}$ \\
\hline
\end{tabular}
bscube2b. dat Refractive Index: 1.515080

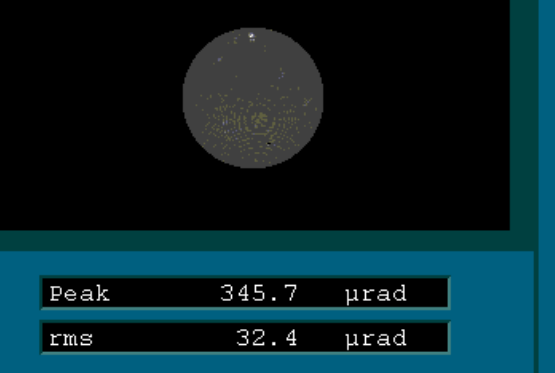




\section{Software}

- Berkeley National Labs - provided software

- 3 beams - 1 beam +2 references - being adapted to new detector for multiple beams

- Tests are underway to check the speed of readout \& processing- $0.5 \mathrm{fps}$ for full frame of $4872 \times 3248-1.3$ fps for partial frame of $4872 \times 800$

- Preliminary stability check

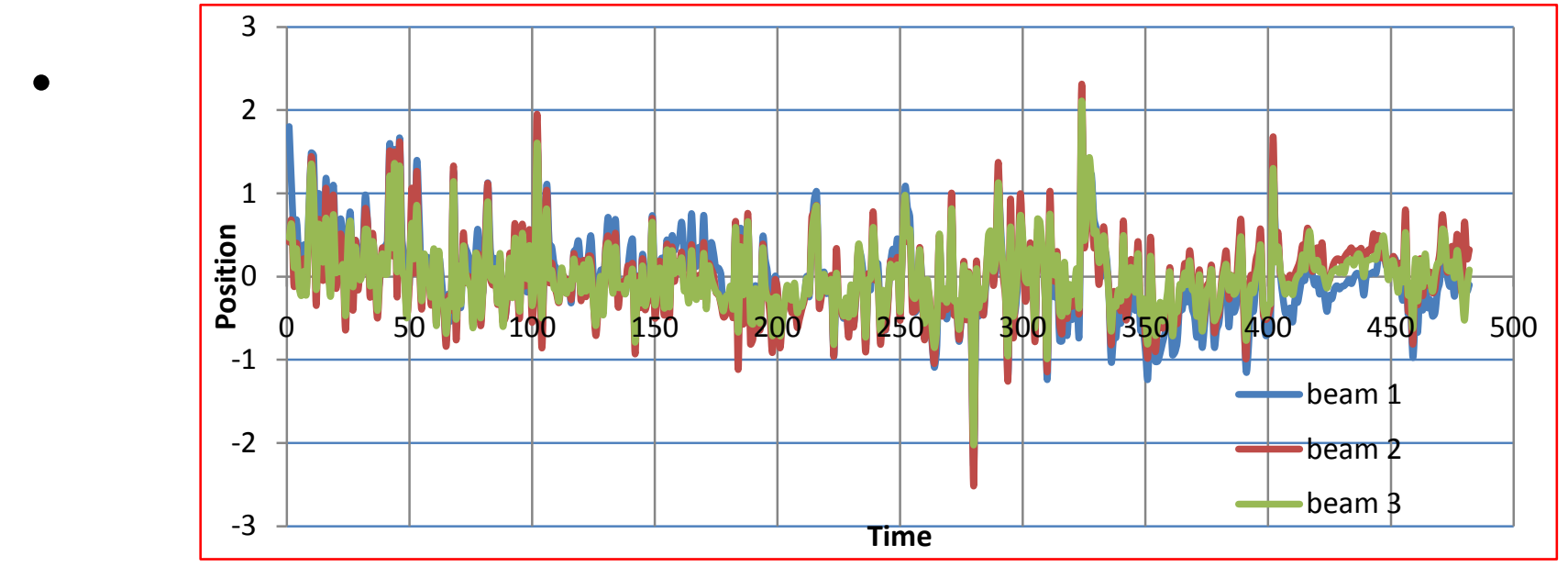




\section{Future work}

- Immediate work

$\checkmark$ Replace detector

$\checkmark$ Etalon test \& analysis

$\checkmark$ Detector software

- Assemble components

- Test measurements 
Acknowledgements

Berkeley National Labs providing software 